(C2008 IEEE. Personal use of this material is permitted. However, permission to reprint/republish this material for advertising or promotional purposes or for creating new collective works for resale or redistribution to servers or lists, or to reuse any copyrighted component of this work in other works must be obtained from the IEEE 


\title{
3G Wireless Communications for Mobile Robotic Tele-Ultrasonography Systems
}

\author{
Saleem Garawi, Robert S. H. Istepanian, and Mosa Ali Abu-Rgheff
}

\begin{abstract}
Mobile healthcare (m-health) is a new paradigm that brings together the evolution of emerging wireless communications and network technologies with the concept of 'connected healthcare' anytime and anywhere. In this article, we present the performance analysis of an end-to-end mObile Tele-Echography using an ultra-Light rObot (OTELO), over the third-generation $(3 \mathrm{G})$ mobile communications network. The experimental setup of the OTELO system over a $3 \mathrm{G}$ connectivity link used to measure the system performance is described. The performance of the relevant medical data and the relevant quality of service (QoS) issues defined in terms of the average throughput, delta-time packet delay, and jitter delay are investigated. The real-time $3 \mathrm{G}$ performance results show the successful operation of this bandwidth demanding robotic m-health system.
\end{abstract}

\section{INTRODUCTION}

M-Health has been defined as "mobile computing, medical sensor, and communications technologies for healthcare" [1]. This emerging concept represents the evolution of e-health systems from traditional desktop "telemedicine" platforms to wireless and mobile configurations. Current and emerging developments in wireless communications integrated with developments in pervasive and wearable technologies will have a radical impact on future healthcare delivery systems. One of the new areas of advanced mobile healthcare applications that has not been explored and investigated in detail is the wireless robotic tele-ultrasonography (U.S.) system.

It is well known clinically that ultrasound scanning is a well-established noninvasive method that is easy to use and very well adapted for routine clinical examinations in specialist medical centres and hospitals. However, most of the available portable ultrasonography and existing ultrasonography systems require the expert to carry out the examination on site. Although these systems offer quick and reliable noninvasive diagnosis in many clinical scenarios, the major drawback of these portable ultrasound systems is that they are not available in small medical centres, isolated sites, and rescue vehicles in emergency cases. Their usefulness is dependent on the operator's (expert) skills. In such circumstances, robotic tele-ultrasonography could be useful. In addition, such robotic telemedicine systems could be very valuable for training in nonspecialist sonograph remote medical centres, and can also be valuable for expert opinion in combat and military scenarios as well.

The wider availability of $3 \mathrm{G}$ systems in most of the European and developing countries will inevitably allow the wider use of such wireless robotic m-health systems, especially in remote and isolated areas, which will certainly reflect on better healthcare efficiency and improved medical care in these countries.

One of the first ultrasound telemedicine studies on remote examinations was reported in the mid-1990s, where the ultrasound video images acquired by the technician at the patient's side were transmitted to a medical expert [2]. However, these systems were not efficient enough for proper medical validation because of their 'expert dependency' on the relevant ultrasound examination.

In 1998, videoconferencing was used between two experts, with one of them was performing the echography examination [3]. Both experts could simultaneously discuss the obtained ultrasound image, and the expert who was distant from the patient could suggest a different probe orientation to his peer for better observation and analysis of the area of interest. In 2000, the European project TeleInVivo was developed [4], in which the echography was performed by a clinical expert standing next to the patient, then ultrasound data were sent via satellite to a data base station and processed to reconstruct a $3 \mathrm{D}$ representation of anatomical regions of interest [5].

In Japan, tele-operated robots have been set up to perform a remote ultrasound examination between two nearby sites with terrestrial communications [6, 7].

All these studies have shown the necessity of a skilled ultrasound expert to drive the robotic structure holding the probe. We have developed a new generation of specific lightweight, 


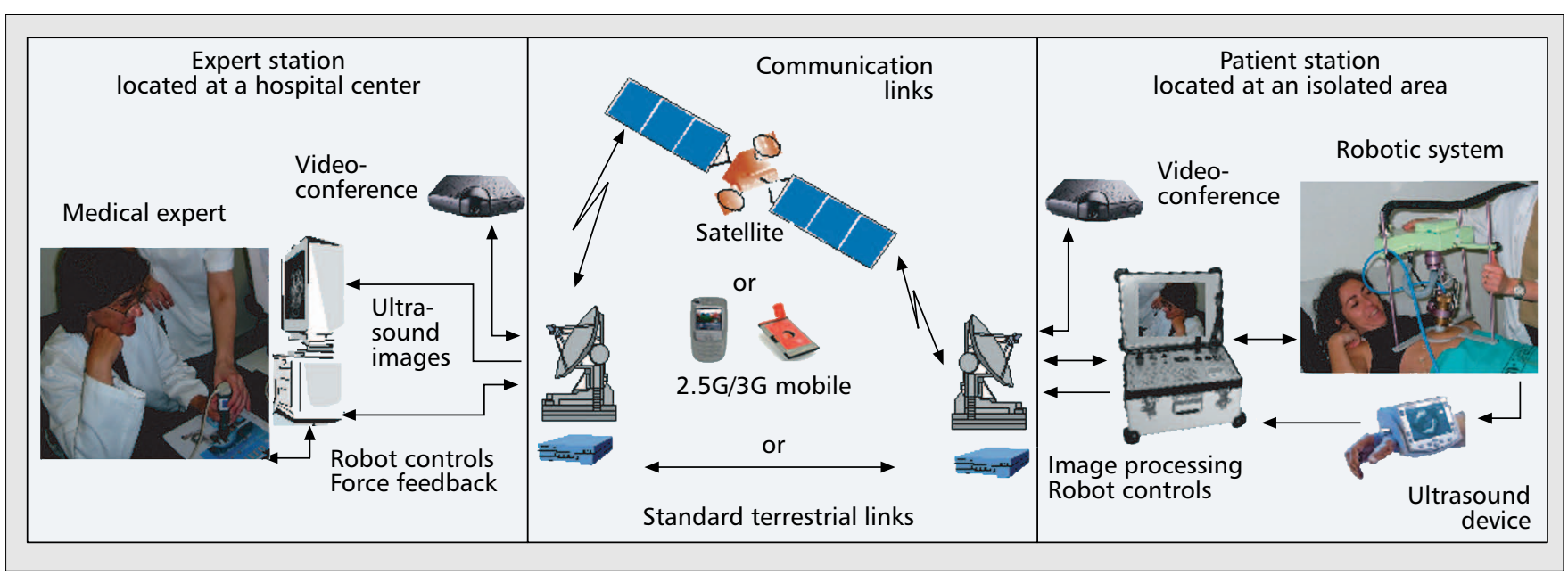

Figure 1. The OTELO mobile robotic system, general block diagram, and different communication links.

portable, and fully integrated robotic devices for tele-ultrasound. These robotic devices have different degrees of freedom (DoFs) dedicated to special applications. The number of degrees in the robotic head or arm represents the number of movements, and a flexibility that translates as close as possible to human hand movement, for example, 6 DoF represents movements in total $\mathrm{X}, \mathrm{Y}, \mathrm{Z}$ and diagonal directions that the human hand can do.

The article is structured as follows. The following section presents an overview of the OTELO system. Next, we outline the $3 \mathrm{G}$ wireless connectivity of the system and the medical data-rate requirements. The article then presents the experimental setup, and goes on to present the performance analysis and discuss the results. The final section presents the conclusions of the article.

\section{G MOBILE ROBOTIC TELE-ECHOGRAPHY SYSTEM}

The advanced medical robotic system, mObile Tele-Echography using an ultra-Light rObot (OTELO), was a European Information Society Technologies (IST) funded project that developed a fully integrated end-to-end mobile teleechography system for population groups that are not served locally, either temporarily or permanently, by medical ultrasound experts. It comprises a fully portable tele-operated robot allowing a specialist sonographer to perform a real-time robotised tele-echography (ultrasonography) to remote patients [7]. The system comprises three main parts:

- The expert station: where the medical expert interacts with a dedicated patented pseudohaptic fictive probe instrumented to control the positioning of the remote robot and emulates an ultrasound probe that medical experts are used to handle, thus providing better ergonomy.

-The communication links: OTELO is adaptable to operate on different types of communication (satellite, $3 \mathrm{G}$ wireless and terrestrial) links. In this article, we address the performance of the system under $3 \mathrm{G}$ mobile connectivity. (The other links have been addressed elsewhere $[5,7,8]$; these different communication links will allow the universal usage of the system based on the availability of these technologies in different geographical locations.)

- The patient station: composed of a $6 \mathrm{DoF}$ lightweight robotic system and its corresponding control unit. This robot manipulates an ultrasound probe according to orders sent by the medical expert. The probe also allows the grabbing of the ultrasound images that are sent back to the expert. Figure 1 shows the general end-toend functionality of the OTELO system.

Three types of critical data are to be transmitted over the OTELO system: robotic control data, ultrasound still images, and medical ultrasound streaming data. In this article, we only address the performance issues regarding the controlled ultrasound medical streams, since this type of medical data represents the most "demanding data-rate" requirements of such robotic telemedicine systems.

The robotic system has also a force feedback mechanism in order to allow the expert to move the fictive probe and control the distant probeholder for the remote robotic system.

We observed that the voice packet data takes priority and could cause some degradation (in terms of the packet delays and jitter) on the ultrasound reception. In addition, during the "ultrasound scan" voice is rarely needed by either the patient or the expert. Hence, we consider that both the ambient video plus the voice (videoconference) can be transmitted when such a data type is required following the reception of ultrasound data by the expert station. However, if sufficient $3 \mathrm{G}$ bandwidth is available, simultaneous ultrasound stream and videoconferencing data transmission can be accommodated. It is reported that at least 80 percent of these in-vivo tests have led to comparable results with a conventional ultrasound examination for the organs examined and detected [7, 8].

It is well known that $3 \mathrm{G}$ wireless technologies present an enhanced mobile platform for many wireless telemedicine applications $[8,9]$; in general, for: 


\begin{tabular}{|c|c|c|c|}
\hline Medical data & Data description & $\begin{array}{l}\text { Data rates }(\mathrm{kb} / \mathrm{s}) \\
\text { and resolution }(\mathrm{dB})\end{array}$ & $\begin{array}{l}\text { Data flow direction, patient-to-expert } \\
\text { (P-to-E), expert-to-patient (E-to-P) }\end{array}$ \\
\hline Still US images & Grayscale, $512 \times 512$ pixels & 14-97 kbytes & Uplink, P-to-E \\
\hline Stream US images & Grayscale, CIF (R.O.I.), $200 \times 200$ pixels & 10 frames $/ \mathrm{s},>35 \mathrm{~dB}$ & Uplink, P-to-E \\
\hline Stream US images & Grayscale, CIF, $352 \times 288$ pixels & 7 frames $/ \mathrm{s},>35 \mathrm{~dB}$ & Uplink, P-to-E \\
\hline Stream US images & QCIF, $176 \times 144$ & 5 frames $/ \mathrm{s},>36 \mathrm{~dB}$ & Up and down, P-to-E and E-to-P \\
\hline Videoconf. option 1 & QCIF, $176 \times 144$ & 7.5 frames $/ \mathrm{s}$ & Up and down, P-to-E and E-to-P \\
\hline
\end{tabular}

- High mobility: 3G offers a data rate of 144 $\mathrm{kb} / \mathrm{s}$ for rural outdoor mobile use for a user traveling at a speed of more than $120 \mathrm{~km}$ per hour.

- Low mobility: $3 \mathrm{G}$ offers a data rate of 384 $\mathrm{kb} / \mathrm{s}$ downlink for pedestrian users traveling at a speed of less than $5 \mathrm{~km}$ per hour ( and up to $2 \mathrm{Mb} / \mathrm{s}$ indoors).

These data rates enables $3 \mathrm{G}$ connectivity to reach anyone, anywhere and transmit any kind of information in real time. $3 \mathrm{G}$ may provide the following services:

- Videoconferencing: to permit videophonetype communication between mobile terminals.

- Video streaming: video recording and images of various kinds can be sent and transmitted with this service. Customers will thus be able to receive real-time television programs on their $3 \mathrm{G}$ terminals for entertainment, cultural, or educational purposes.

- Internet browsing: the user can browse on the Web directly with the mobile terminals.

- Application sharing: applications running on the customer terminal can use processing resources resident on a remote server (e.g., one managed by the service provider).

The selected U.S. streams will be transmitted over different available $3 \mathrm{G}$ network data rates. These range from 56 to $384 \mathrm{~kb} / \mathrm{s}$ (uplink). These rates are specifically related to the patient-station uplink that represents the specific communication bottleneck of this telemedicine $3 \mathrm{G}$ connectivity channel. The patient station sends ultrasound images, ultrasound streams, ambient video, sound, and robot control data, while it receives only robot control, ambient video, and sound from the expert station (i.e., expert-station uploading).

The classification of the OTELO traffic is mapped to the three major traffic classes defined by the Third Generation Partnership Project (3GPP) for Universal Mobile Telecommunication System (UMTS) quality of service (QoS) classes. The best-suited QoS class for video streaming is service class "Streaming" which preserves the time relation (variation) between information entities of the stream. However, for

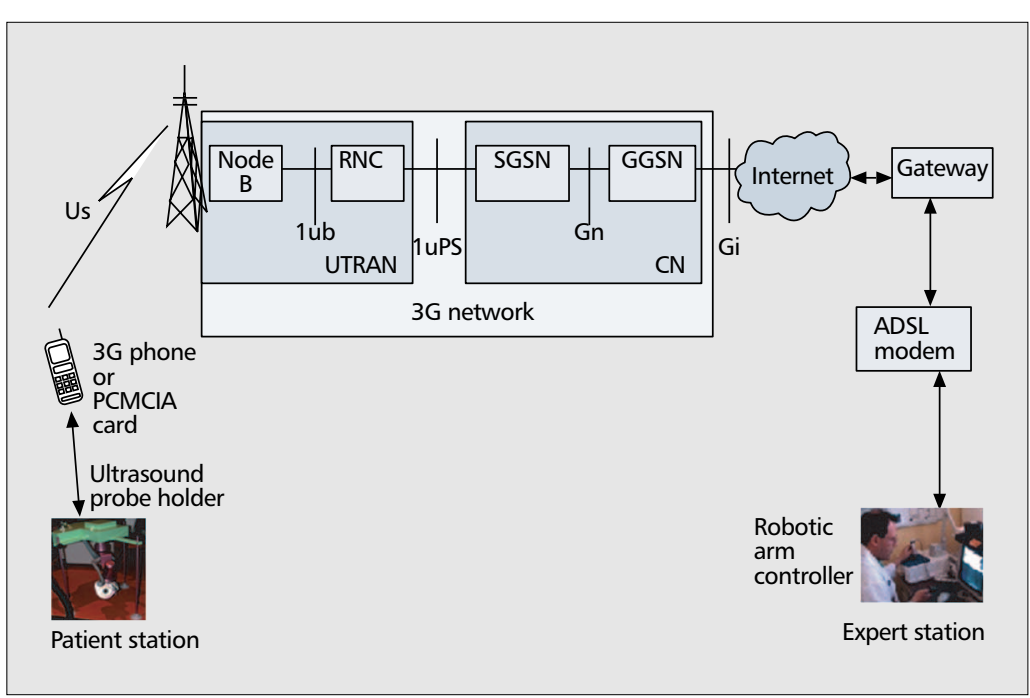

Figure 2. OTELO mobile robotic system connectivity over a $3 G$ network.

medical image sequences with real-time (RT) requirements, the "Conversational" class would be necessary.

The general functional modalities of the system are given in Table 1, with those addressed in this article shown in boldface letters.

\section{EXPERIMENTAL SETUP ENVIRONMENT}

The experimental setup is designed to measure the end-to-end system performance over the $3 \mathrm{G}$ network, as shown in Fig. 2.

The following performance metrics are measured:

- Average throughput (ultrasound stream and robot control data)

- End-to-end packet delay and delay jitter.

The ultrasound scanner data is acquired at the rate of 13 frames/s, each frame with the resolution of $(320 \times 240)$ pixels for the videoconferencing format, and $(176 \times 144)$ pixels for the Quarter Common Intermediate Format (QCIF). The robotic data flow bursts from the expert station at 16 bytes payload on $70 \mathrm{~ms}$ time interval, 
and the received robot data stream from the patient station is updating the robotic head position continuously.

The patient station is connected to a $3 \mathrm{G}$ terminal via a wireless card connected to a laptop PC. The tests were carried out at different network loading conditions (especially at peak working hours) and the presented results reflect these network conditions.

\section{IMPLEMENTATION OF THE Streaming Protocol}

It is well known that protocols for streaming media are commonly designed and standardized

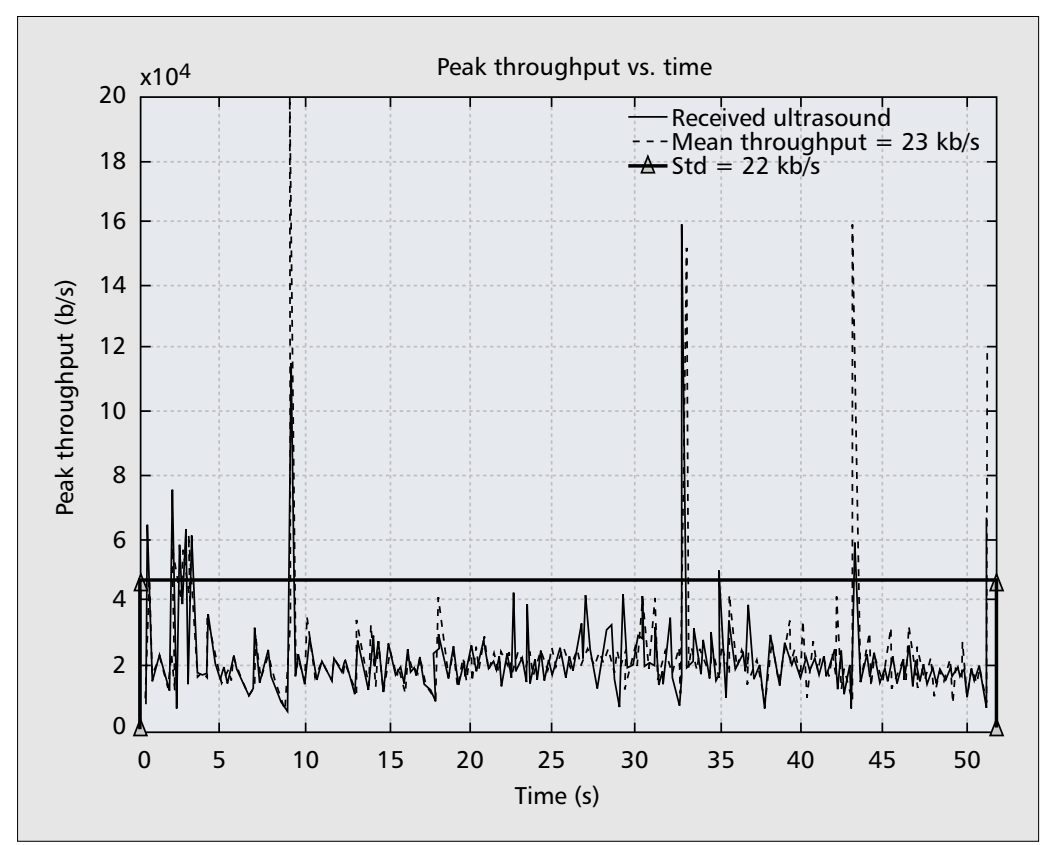

Figure 3. Sample peak throughput of the received ultrasound stream by the expert station.

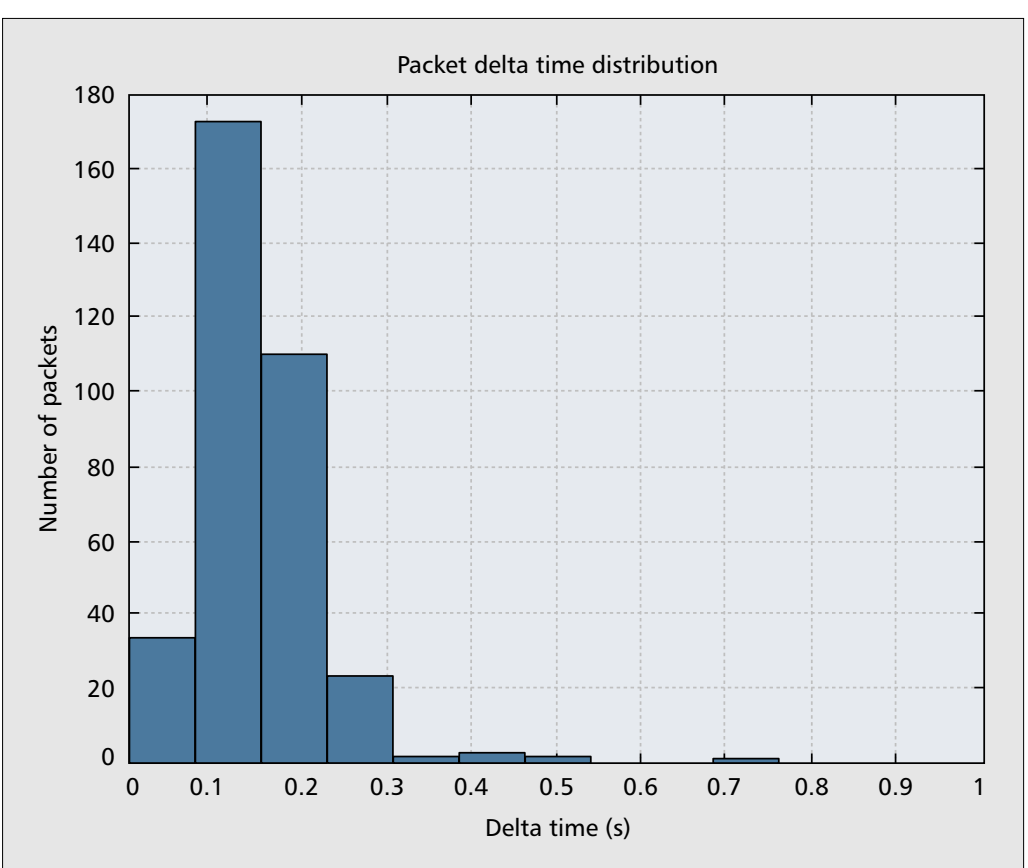

Figure 4. Distribution of the delta time packet delay at the expert station. for communications between clients and streaming servers. They are concerned with issues such as network addressing, transport, and session control [10]. The transport protocol family for media streaming includes User Datagram Protocol (UDP), Transmission Control Protocol (TCP), Real-Time Protocol (RTP), and RealTime Transport Control Protocol (RTCP).

Since TCP retransmission introduces delays that are not acceptable for real-time streaming applications with stringent delay requirements, especially for transmission over fading wireless links, UDP is typically employed as the transport protocol for video streams over such fading channels.

RTP is an Internet standard protocol designed to provide end-to-end transport functions for supporting real-time applications. RTCP is a companion control protocol with RTP and is designed to provide QoS feedback to the participants of an RTP session; therefore, the RTP/UDP/IP protocol is applied in our work. The UDP/IP protocol is used for the robot data that is transmitted in both directions. Although the effect of packet loss on the robotic control could affect the mechanical functionality of the robotic control system, the tests carried out on the OTELO system with an uplink of (64 $\mathrm{kb} / \mathrm{s}$ ) have shown the reliable functioning of the robotic system in the patient station with the minimal packet loss of ( $<0.5$ percent $)$.

\section{Ultrasound StREAM CODEC}

The video compression standard used in this application is $\mathrm{H} 263$ codec, which has a wide range of applications, including medical consultation and diagnosis at a distance. The H.263 is aimed particularly at video coding for low bit rates (typically, 20 to $30 \mathrm{~kb} / \mathrm{s}$ and above). Further details on this codec design and the imaging functionality of this codec for OTELO can be found in [11].

\section{Performance Results And DISCUSSION}

The OTELO system was tested on a live $3 \mathrm{G}$ network (Vodafone, U.K.). The experimental tests for the system were carried within the greater London area between Kingston University, London (patient side) and St. George's Medical School, London (expert side). The following results summarize some of the performance tests carried out.

\section{ULtRASOUND ThROUGHPUT ANALYSIS}

A sample of the instantaneous throughput of the ultrasound stream captured by the expert is shown in Fig. 3, where the average throughput obtained was $23 \mathrm{~kb} / \mathrm{s}$ and the standard deviation (std) value of $22 \mathrm{~kb} / \mathrm{s}$ represented the patient station uplink average throughput value.

The variation in the throughput shown in the figure can be attributed to the movement of the robotic probe holder in addition to the variations in the network conditions.

The number of stream packets taken for the analysis was 350 . This stream length was selected based on the experimental $3 \mathrm{G}$ test results on 
OTELO, where this stream length represents a good average measure for the performance results of the system for a complete ultrasound scan session that can last, in general, between two and four minutes. These tests also indicated that approximately 82 percent of the packets received at the expert station were at rate of $18.5 \mathrm{~kb} / \mathrm{s}$, whilst the reminder of the packets were received at data rates of up to $60 \mathrm{~kb} / \mathrm{s}$.

The performance test of the system in terms of packet loss indicated that none of the 360 RTP packets captured by the expert side, in good network conditions, was lost; whereas in a different network (congested) condition test, the result indicated that only 2 out of 1192 RTP packets ( 0.17 percent) captured were lost.

\section{Delay Distribution}

The delta-time distribution of the stream packets is shown in Fig. 4, where the performance analysis of the stream packet delay at the expert station gives an average packet delta time (time difference between two consecutive packets) of $0.148 \mathrm{sec}$.

The delay distribution shows a maximum delay of around $0.3 \mathrm{sec}$ for just about 7 percent of the received packets, while 50 percent and about 30 percent of the packets show delays of around 0.12 and $0.22 \mathrm{sec}$, respectively. There are several factors that cause this delay across the wireless link: the delay access on each ON period (variable), the delay of the multiplex process (variable), and the transmission delay. The connection between both system ends is always ON during the entire clinical session [9]. It should also be noted that any video packet that arrives beyond its delay bound (e.g., its play-out time) is useless and can be regarded as lost [10].

The delay variation (jitter) across this system's link is considered a key factor for a reliable real-time medical ultrasound stream reception at the expert station. The results also indicate that the delay jitter encountered by the received packets degrades the reliability of the ultrasound images in terms of irregular frames per second (fps) production.

\section{ROUND-TRIP TIME DELAY}

Generally, the network RTT can be defined as the time it takes to transmit one packet from, say, a server to a terminal plus the time it takes for the corresponding packet to be sent back from the terminal to the server [12]. The formula for the RTT delay regarding OTELO system is represented as follows:

Expert side generates and sends robot control (Expert-Patient link delay)

+ Patient station responds by transmitting ultrasound stream of images (Patient-Expert link delay).

Different packet sizes of 100, 200, 300, 500, 1000 , and 1400 bytes are tested to characterize various delay performances. These packet sizes are chosen to cover the possible size range of the packets generated by the $\mathrm{H} 263$ codec used by the system.

Internet Control Message Protocol (ICMP) is

\begin{tabular}{|c|c|c|c|c|c|c|c|}
\hline \multicolumn{8}{|c|}{$\begin{array}{l}\text { Packet sizes (bytes) } \\
\text { Ultrasound and robot }\end{array}$} \\
\hline & 100 & 200 & 300 & 500 & 1000 & 1400 & 16 \\
\hline $\mathrm{RTT}$ (ms) & 206 & 257 & 297 & 360 & 521 & 638 & 100 \\
\hline
\end{tabular}

Table 2. Comparative RTT results of different ultrasound stream and robotic packet sizes.

used for pinging the expert station from the patient station at 500 to $1000 \mathrm{~ms}$ time intervals. ICMP provides some error-detection mechanisms and it can be used to send error messages or other messages for network diagnosis [12]. The RTT delay test is performed with patient-station data rates of $256 \mathrm{~kb} / \mathrm{s}$ for downlink and up to $64 \mathrm{~kb} / \mathrm{s}$ for the uplink channel. Table 2 summarizes the comparative results of the RTT values for different ultrasound packet sizes as well as the robotic control data size of 16 bytes. These results indicate the end-to-end RTT of the path defined above.

The test results also show that the latencies of the $3 \mathrm{G}$ wireless link of the system are the highest (around 80 to 90 percent) of the total end-to-end delay.

The end-to-end delay of the system is based on the average packet delta time results, as illustrated in Table 2, which is closely correlated (RTT/2) to pinging 300 bytes (boldface results in Table 2) from the patient station to the expert station that is used as an average ultrasound transmitted packet size. Based on the above, the maximum delay of probe movement to received image (expert-patient-expert) path was found to be around $325 \mathrm{~ms}$ under these specific ultrasound-encoding conditions.

\section{CONCLUSIONS}

The experimental test results for transmitting ultrasound streams encoded in the (QCIF) format using the H.263 codec have demonstrated successful transmission in $3 \mathrm{G}$ real-time environments. The quality of the received ultrasound information was $5 \mathrm{fps}$ with an objective quality measure of $35 \mathrm{~dB}$ peak signal to noise ratio (PSNR). These values represent the minimum bounds that are clinically acceptable by the medical experts using the OTELO system for prediagnosis requirements of such $3 \mathrm{G}$ mobile robotic telemedicine systems. These results are achieved using $64 \mathrm{~kb} / \mathrm{s}$ at the patient station uplink. Enhanced performance can be achieved using higher rates and depending on the $3 \mathrm{G}$ network operators channel assignments.

We have also found that network delay jitter variations were still within the acceptable boundaries of maintaining high-quality realtime interaction for the system; $297 \mathrm{~ms}$ compared to a maximum delay of $325 \mathrm{~ms}$. In general, we can conclude that such advanced mobile robotic telemedicine systems can successfully provide clinically acceptable quality ultrasound data using commercial $3 \mathrm{G}$ networks. 


\section{ACKNOWLEDGMENTS}

The quality of the received ultrasound information was

5 fps with an objective quality measure of $35 \mathrm{~dB}$ PSNR. These values represent the minimum bounds that are clinically acceptable by the medical experts. project.

\section{REFERENCES} TX, June 1995, pp. 292-99.
We are grateful to the European Union for support for this project (EU IST-2001-32516 project "OTELO: Integrated, end-to-end, mobile teleechography system"). The authors are also grateful to Vodafone U.K. for their support to this

[1] R. S. H. Istepanian, E. Jovanov, and Y. T. Zhang, "Mhealth: Beyond Seamless Mobility for Global Wireless Healthcare Connectivity-Editorial," IEEE Trans. Info. Tech. in Biomed., vol. 8, no. 4, Dec. 2004, pp. 405-14.

[2] J. Sublett, B. Dempsey, and A.C. Weaver, "Design and Implementation of a Digital Teleultrasound System for Real-Time Remote Diagnosis," Comp.-Based Med. Sys.,

[3] R. Ribeiro et al., "Teleconsultation for Cooperative Acquisition, Analysis and Reporting of Ultrasound Studies," TeleMed '98, London, U.K., 25-26 Nov. 1998.

[4] G. Kontaxakis, S. Walter, and G. Sakas, EU-TelelnViVo, "An Integrated Portable Telemedicine Workstation Featuring Acquisition, Processing and Transmission Over Low-Bandwidth Lines of 3D Ultrasound Volume Images," Info. Tech. Apps. in Biomed. 2000, U.S., Nov. 2000.

[5] A. Vilchis et al., "Robotic Tele-Ultrasound System (TER): Slave Robot Control," 1st IFAC Conf. Telematics App. in Automation and Robotics, Weingarten, Germany, 24-26 July 2001, pp. 95-100.

[6] K. Masuda et al., "Development of Remote Echographic Diagnosis System by Using Probe Movable Mechanism and Transferring Echogram via High Speed Digital Network," Proc. IX Mediterranean Conf. Med. and Biological Eng. and Comp., Pula, Croatia, June 2001, pp. 96-98.

[7] F. Courreges et al., "Clinical Trials and Evaluation of a Mobile, Robotic Tele-Ultrasound System," J. Telemed. and Telecare, suppl. 1, 2005, pp. 46-49.

[8] S. A. Garawi et al., "Performance Analysis of a Compact Robotic Tele-Echography E-Health System over Terrestrial and Mobile Communication Links," Proc. 5th IEE Int'l. Conf. 3G Mobile Commun. Tech., London, U.K., 18-20, Oct. 2004, pp.118-22.

[9] H. Holma and A. Toskala, Eds., WCDMA for UMTSs, Wiley, 2000

[10] W. Dapeng et al., "Streaming Video over the Internet: Approaches and Directions," IEEE Trans. Circuits and Sys. for Video Tech., vol. 11, no. 3, Mar. 2001.

[11] R. Karel, "H.263: Video Coding for Low-Bit-Rate Communication," IEEE Commun. Mag., Dec. 1996, pp. 42-45.

[12] M. Taferner and E. Bonek, Wireless Internet Access over GSM and UMTS, Springer-Verlag, 2002.

\section{BIOGRAPHIES}

SALEM GARAWI (a.salem@kingson.ac.uk) received his B.S.C. degree in electrical and electronic engineering from the University of South Banks London, United Kingdom, in 2000. He is currently finalizing his Ph.D. studies in $3 G \mathrm{~m}$ health systems in the MINT Centre at Kingston University, London. Previously he worked in several U.K. and overseas industrial and academic institutions.

ROBERT S. H. ISTEPANIAN [M'94, SM'97] received a Ph.D. degree in electronic and electrical engineering from Loughborough University, United Kingdom, in 1994. From 1984 to 1998 he worked in different overseas industrial and academic positions. In 1988 he was a visiting research fellow with the Department of Electronic and Electrical Engineering, Loughborough University. He was with the same department from 1994 to 1995 as a postdoctoral research fellow. From 1996 to 1999 he was a senior lecturer at the University of Portsmouth, United Kingdom. In 1999 he was an sssociate professor at the Universities of Western Ontario and Ryerson, Toronto, Canada. He became head of the Mobile Information Engineering and E-Med Systems research group in the Department of Electronic and Computer Engineering, Brunel University, West London, United Kingdom, in 1999. In early 2003 he joined the School of Computing, Information Systems and Mathematics, Kingston University, as a professor of data communications and founding director of the Mobile Information and Network Technologies (MINT) Research Centre. He is also a visiting professor at St. George's Medical School, University of London. He is a Fellow of the Institute of Electrical Engineers. He has also served on numerous technical committees for the IEEE, EMBS, and other international conferences. He currently serves on the editorial boards of IEEE Transactions on Information Technology in Biomedicine and IEEE Transactions on Nanobioscience, and was one of the founding special area editors of IEEE Transactions on Information Technology in Biomedicine, on Telemedicine and e-Health, in 1997.

MoSA ALI ABU-RGHEFF [SM] received a B.S.c with honours from Leeds University, United Kingdom, and a Ph.D. in communications engineering from Bradford University, United Kingdom. After graduation he held a number of industrial posts in research and development. He joined the University of Bradford in 1990 and moved to the University of Plymouth in 1991. He has authored numerous publications in journals and international conference proceedings on modulation/coding for digital communications. His current research interests include mobile communications and mobility management. He is leading mobile communications research in the Faculty of Technology, University of Plymouth. He is a Chartered Engineer (U.K.), and is a member of IEE, and national and international technical/advisory committees. 